\title{
Intensity of Physical Activity and Depressive Symptoms in College Students: Fitness Improvement Tactics in Youth (FITYou) Project
}

This article was published in the following Dove Press journal: Psychology Research and Behavior Management

\author{
Feng Guo' \\ Ying Tian (iD ${ }^{2}$ \\ Fei Zhong ${ }^{3}$ \\ Chunchun Wu (iD ${ }^{3}$ \\ Yufei Cui ${ }^{\prime}$ \\ Cong Huang $\mathbb{D}^{3,4}$ \\ 'Institute of Exercise Epidemiology and \\ Department of Physical Education, \\ Huaiyin Institute of Technology, Huai'an, \\ People's Republic of China; ${ }^{2}$ College of \\ Sports Science, Shenyang Normal \\ University, Shenyang, People's Republic of \\ China; ${ }^{3}$ Department of Sports and \\ Exercise Science, College of Education, \\ Zhejiang University, Hangzhou, People's \\ Republic of China; ${ }^{4}$ Department of \\ Medicine and Science in Sports and \\ Exercise, Tohoku University Graduate \\ School of Medicine, Sendai, Japan
}

Purpose: To investigate whether the physical activity at different intensities is correlated with depressive symptoms in college students.

Patient and Methods: This cross-sectional study was a part of the Fitness Improvement Tactics in Youth Project, which was conducted in 2017. A total of 2,820 college freshmen aged 15-24 years were included in this study, of whom 699 (24.8\%) were males and 2,121 (75.2\%) were females. Depressive symptoms were measured using the Self-rating Depression Scale. Physical activity was assessed using the International Physical Activity Questionnaire-Short Form. Covariates included sociodemographic, lifestyle-related, and health-related factors.

Results: A total of 560 (19.9\%) college students had depressive symptoms. Total physical activity levels (METs $\bullet$ hour/week) were positively associated with frequency of breakfast intake $(p=0.050)$ but no other participant characteristics. Logistic regression analysis showed that the prevalence of depressive symptoms in the highest quartile of physical activity was lower than in the lowest quartile when adjusted for sex, age, ethnicity, only child, smoking status, alcohol use, breakfast frequency, daily sleep duration, body mass index, grip strength, and the number of metabolic syndrome components (odds ratios [95\% confidence intervals $(\mathrm{CI})]: 0.75$ [0.58, 0.98], $p=0.036$ ). Furthermore, high physical activity levels of low-intensity tended to moderately correlate to lower prevalence of depressive symptoms (odds ratios [95\% CI]: Q1, 0.71 [0.55, 0.91], Q2, 0.77 [0.59, 1.01], Q3, 0.75 [0.57, $0.98], p$ for trend $=0.037$ ). Associations of moderate and vigorous physical activity with depressive symptoms were not found. For secondary outcomes, sex-stratified analysis showed that similar findings were only found in females, but not males.

Conclusion: This study indicated that total physical activity and low-intensity physical activity were inversely associated with depressive symptoms in Chinese college students. All these observations showed gender differences.

Keywords: physical activity intensity, mental health, Chinese youth, cross-sectional study

\section{Introduction}

Depressive disorders are the most common mood disorders in young adults aged 18-35 years. ${ }^{1}$ The main clinical characteristics of depression are sadness, loss of interest, low sense of self-worth, slow response, lack of sleep or appetite, poor concentration, and severe fatigue. ${ }^{2}$ Depression can seriously damage an individual's ability to work, learn or cope with daily life. As depression becomes more severe, it may lead to suicide. ${ }^{3}$ For the youth, who aged between 15 and 24 years, ${ }^{4}$ college
Institute of Exercise Epidemiology and

Department of Physical Education, Huaiyin

Institute of Technology, Huai'an 223003 ,

Cong Huang

Department of Sports and Exercise Science, College of Education, Zhejiang University,

I48 Tianmushan Road, Hangzhou 310007,

People's Republic of China,

Email cohuang@zju.edu.cn 
life is an important period in their life because they face great pressure due to academic rigour, economic stress, interpersonal relationship and future employment. ${ }^{5}$ Data indicated that depressive symptoms are correlated with an increased prevalence of cardiovascular diseases and allcause mortality in Chinese adults. ${ }^{6}$ In China, the lifetime prevalence of depression was $6.9 \%$, the prevalence of depression was higher in female, and those individuals with aged 18-34 years had a higher prevalence of depression than adults aged 35-49 years. ${ }^{1}$ However, a metaanalysis showed that the incidence rate of depressive symptoms was $23.8 \%$ among 32,694 Chinese university students from 1997 to $2015 .^{7}$ This meta-analysis also revealed that depressive symptoms among medical students is worse than non-medical students for Chinese college students. ${ }^{7}$ These findings suggested that effective preventive strategies are of great significance in lowering the prevalence of depressive symptoms in youth.

Some studies have reported that depressive symptoms were affected by some factors, such as higher levels of smartphone use, ${ }^{8}$ social media use, ${ }^{9}$ and misperceptions of weight, ${ }^{10}$ in college students. Moreover, fewer outdoor activities and physical activities, short duration and poor quality of sleep, and less breakfast frequently were also associated with higher depressive symptoms scores in Chinese college students. ${ }^{11}$ It is important to note that increasing evidence has shown that lifestyle interventions targeting physical activity can improve depressive symptoms in youth. Specifically, a study of 11,110 adolescents from 10 European countries showed that the frequency of physical activity was negatively correlated with depressive symptoms. ${ }^{12}$ In addition, a study of 2,444 Chinese adolescents showed that the more physical activity performed per week, lower the prevalence of depressive symptoms. ${ }^{13}$ So far, however, few studies have analyzed the association between exercise intensity and depressive symptoms. Tsujita et al suggested that the lower levels of physical activity could be associated with higher levels of depressive symptoms, including daily steps, duration and amount of moderate to vigorous-intensity physical activity in healthy young adults. ${ }^{14}$ A longitudinal study of 8 weeks showed high-intensity interval training and sprint interval training similarly improved depressive symptoms in 36 healthy women. ${ }^{15}$ Besides, a 6-week intervention study showed both high-intensity interval training and moderate continuous training can reduce depressive symptoms in university students. ${ }^{16}$ Moreover, a randomized controlled trial of 18 sessions indicated that high-intensity interval training can improve depression, activity states, cardiovascular functions compared to conventional moderate-intensity continuous training in 44 patients with myocardial infarction. ${ }^{17}$ Also, an 8-week aerobic exercise of moderate-intensity could lower depressive symptoms among individuals with major depressive disorder aged 18 to 30 years. ${ }^{18}$ These experimental studies revealed a causal relationship between exercise and depressive symptoms in youth.

Although these previous studies have demonstrated the association between physical activity intensity and depressive symptoms, further study is needed due to limited and inconsistent evidence. Therefore, the purpose of this study is to investigate whether physical activity intensity is associated with depressive symptoms in Chinese college students.

\section{Patients and Methods}

\section{Study Participants}

We utilized baseline data from the Fitness Improvement Tactics in Youth (FITYou) project for the current study. FITYou aimed to clarify the impact of socioeconomic, lifestyle, physiological status, and metabolic regulation on the health-related quality of life and health status of college students. The project was carried out in September 2017 in Shenyang, China. ${ }^{19,20}$ A total of 4,717 college students were invited to participate in the project, of which 4,323 students provided written informed consent for data analysis (response rate $=91.6 \%$ ). Additional written informed consent from a parent or legal guardian was required for participants under 18 years of age.

Of the participants, data on health checkups from 218 students were not available, 436 had missing depressive symptoms data, and 684 students were missing physical activity data. Among the remaining 2,985 students, data on sex $(n=3)$, age $(n=32)$, only child status $(n=7)$, smoking status $(n=14)$, drinking status $(n=27)$, breakfast frequency $(\mathrm{n}=18)$, sleep duration $(\mathrm{n}=38)$, body mass index $(\mathrm{BMI})$ $(n=6)$, and Metabolic syndrome (MetS) components $(n=20)$ were incomplete. As a consequence, data for a total of 2,820 students were eventually included in the analysis (Figure S1). The study was carried out following the Helsinki Declaration ${ }^{21}$ to protect the rights and personal safety of participants. The research plan was approved by the Ethics Committee of the College of Education, Zhejiang University.

\section{Assessment of Depressive Symptoms}

The Self-rating Depression Scale (SDS) ${ }^{22}$ was used to evaluate depressive symptoms. The evaluation criteria 
are: "1" indicates no time or very little time, "2" indicates sometimes, " 3 " indicates most of the time, and " 4 " indicates most or all of the time. Added the total score of these 20 questions and multiply by 1.25 to get the standard score, which indicates depressive symptoms while greater than 53 according to the Chinese version of SDS. ${ }^{23}$

\section{Assessment of Physical Activity}

Physical activity was measured by the International Physical Activity Questionnaire-Short Form (IPAQ-SF). Participants were instructed to consider all areas of physical activity during the past week. The questionnaire asked participants to indicate how many days they spent in walking, moderate-intensity and highintensity activities, and how long they spent in these activities daily. ${ }^{24}$ Total physical activity was calculated by metabolic equivalents (METs) hour/week and the calculation formula for the total physical activity per week described as (daily walking hours $\times$ days of walking per week $\times 3.3)+($ daily moderate activity hours $\times$ days of moderate activity per week $\times 4.0$ ) + (daily vigorous activity hours $\times$ days of vigorous activity per week $\times 8.0$ ). Total physical activity levels were divided into quartiles for analysis based on its distribution. According to IPAQ-SF, physical activity including lowintensity (walking), moderate-intensity (moderate activity), and high-intensity (vigorous activity). Physical activity levels in three different intensity were calculated and then divided into quartiles, respectively.

\section{Relevant Covariates}

Covariates included sociodemographic, lifestyle-related, and health-related factors. Potential covariates related to demographics and lifestyle-related factors were collected by self-report questionnaire. Age was calculated based on the date of birth and ethnicity was summarized as Han or other. Lifestyle-related information was acquired through multiple-choice questions, including smoking status (current, former, or never smoking), drinking status (current drinker, non-drinker), breakfast frequency $(<4,4-5,6-7$ times/week), and daily sleep duration ( $<6,6-7,7-8, \geq 8$ hours). Height, weight, and grip strength were measured in health examinations by medical practitioners, and BMI was calculated from these data. The definition of each component of metabolic syndrome was based on the 2009 American Heart Association scientific statement. ${ }^{25}$

\section{Statistical Analysis}

Analysis of variance or $X^{2}$ test for continuous and categorical variables were used to describe the characteristics of participants based on physical activity level. Categorical variables were presented as number and percentages, and continuous variables were presented as means and standard deviations.

Logistic regression analysis was used to obtain a linear trend between physical activity level and depressive symptoms, and data were shown as odds ratios and $95 \%$ confidence intervals (CIs). Covariance analysis includes three statistical models adjusted according to covariates, taking the relationship between gender and physical activity intensity into account. Model 1 adjusted for sex, age, ethnicity, and only child status. Model 2 added lifestyle-related variables including smoking status, alcohol use, breakfast frequency, and daily sleep duration. Model 3 adjusted for model 2 plus BMI, grip strength, and the number of metabolic syndrome components. All tests were two-tailed, and a $p$-value $<0.05$ was considered statistically significant. IBM SPSS Statistics 22.0 software for MAC (IBM Corp, Armonk, NY, USA) was used for the statistical analysis in this study.

\section{Results}

\section{Participants' Characteristics}

Participant characteristics according to physical activity level are summarized in Table 1. A total of 2,820 college freshmen aged 15-24 years were included in this study, of whom 699 (24.8\%) were males and 2,121 (75.2\%) were females. There were no significant differences in BMI, smoking status, alcohol use, sleep duration, and metabolic syndrome components among students with different physical activity levels. The number of people who ate breakfast 6-7 times a week occupied the highest proportion among all levels of physical activity, and the lowest frequency was 4-5 times a week.

\section{Association Between Total Physical Activity Levels and Depressive Symptoms}

Table 2 shows the relationship between physical activity levels and depressive symptoms in participants before and after adjusting for potential covariates. In the univariate model, participants with physical activity at the Q2 level had the lowest prevalence of depressive symptoms, while participants at the Q3 level had relatively more depressive symptoms ( $p$ for trend $=0.008$ ). In general, higher physical activity levels were associated with a lower prevalence of depressive symptoms. 
Table I Participants' Characteristics According to Physical Activity Levels in College Freshmen ( $\mathrm{n}=2820)$

\begin{tabular}{|c|c|c|c|c|c|}
\hline \multirow[t]{2}{*}{ Variable $^{\text {a }}$} & \multicolumn{5}{|c|}{ Quartiles of Total Physical Activity Levels } \\
\hline & QI & Q2 & Q3 & Q4 & $p$ value $^{b}$ \\
\hline Range, METs hour/week & $<27.0$ & $27.0-58.0$ & $58.1-113.0$ & $>113.0$ & \\
\hline Participants, no. (\%) & $699(24.8)$ & $710(25.2)$ & $703(24.9)$ & $708(25.1)$ & \\
\hline Sex (female), no. (\%) & $525(75.1)$ & $547(77.0)$ & $508(72.3)$ & $54 \mid(76.4)$ & 0.164 \\
\hline Age (years) (SD) & I8.I (0.85) & I8.I (0.80) & I8.I (0.75) & $18.1(1.01)$ & 0.550 \\
\hline Han ethnicity, no. (\%) & $586(83.8)$ & $601(84.6)$ & $591(84.1)$ & $584(82.5)$ & 0.727 \\
\hline Only child, yes (\%) & $410(58.7)$ & $410(57.7)$ & $397(56.5)$ & $400(56.5)$ & 0.810 \\
\hline BMI $\left(\mathrm{kg} / \mathrm{m}^{2}\right)(\mathrm{SD})$ & $21.8(4.0)$ & $21.7(3.9)$ & $21.8(4.2)$ & $21.6(3.8)$ & 0.650 \\
\hline \multicolumn{6}{|l|}{ Smoking status } \\
\hline Never, no. (\%) & $674(96.4)$ & $686(96.6)$ & $667(94.9)$ & $683(96.5)$ & \\
\hline Former, no. (\%) & II (I.6) & II (I.5) & $21(3.0)$ & $8(I . I)$ & \\
\hline Current, no. (\%) & $14(2.0)$ & $13(1.8)$ & $15(2.1)$ & $17(2.4)$ & 0.209 \\
\hline Alcohol use (yes), no. (\%) & $76(10.9)$ & $86(12.1)$ & $91(12.9)$ & $93(13.1)$ & 0.557 \\
\hline \multicolumn{6}{|l|}{ Breakfast (times/week) } \\
\hline$<4$, no. $(\%)$ & $155(22.2)$ & $114(16.1)$ & $132(\mid 8.8)$ & $118(16.7)$ & \\
\hline $4-5$, no. (\%) & III (I5.9) & III (I5.6) & $99(14.1)$ & $106(15.0)$ & \\
\hline $6-7$, no. $(\%)$ & $433(61.9)$ & $485(68.3)$ & $472(67.1)$ & $484(68.4)$ & 0.050 \\
\hline \multicolumn{6}{|l|}{ Sleep duration (hours/day) } \\
\hline$<6$, no. $(\%)$ & $173(24.7)$ & $173(24.4)$ & $169(24.0)$ & $168(23.7)$ & \\
\hline 6-7, no. (\%) & $264(37.8)$ & $263(37.0)$ & $259(36.8)$ & $273(38.6)$ & \\
\hline $7-8$, no. (\%) & $192(27.5)$ & $216(30.4)$ & $209(29.7)$ & $203(28.7)$ & \\
\hline$\geq 8$, no. (\%) & $70(10.0)$ & $58(8.2)$ & $66(9.4)$ & $64(9.0)$ & 0.961 \\
\hline Grip strength, kg (SD) & $26.7(7.8)$ & $26.8(7.8)$ & $27.4(8.5)$ & $27.0(8.1)$ & $0.28 \mathrm{I}$ \\
\hline MetS components, no. (SD) & $1.2(0.47)$ & $1.2(0.49)$ & $1.2(0.49)$ & $\mathrm{I} .2(0.42)$ & 0.216 \\
\hline
\end{tabular}

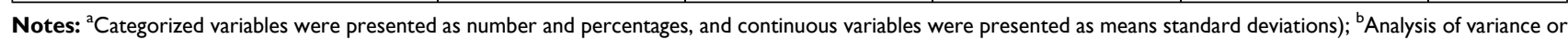
$X^{2}$ test, as appropriate.

Abbreviations: BMI, body mass index; MetS, metabolic syndrome.

Table 2 Association Between Total Physical Activity Levels and Depressive Symptoms in College Freshmen $(n=2820)$

\begin{tabular}{|c|c|c|c|c|c|}
\hline & \multicolumn{5}{|c|}{ Quartiles of Total Physical Activity Levels } \\
\hline & QI, n=699 & Q2, $n=710$ & Q3, $n=703$ & Q4, $n=708$ & $p$ value $^{a}$ \\
\hline Range, METs hour/week & $<27.0$ & $27.0-58.0$ & $58.1-113.0$ & $>113.0$ & \\
\hline Depressive symptoms, no. & 169 & 124 & 137 & 130 & \\
\hline Non-adjusted model & 1.00 (ref) & $0.66(0.5 \mathrm{I}, 0.86)$ & $0.76(0.59,0.98)$ & $0.7 I(0.55,0.91)$ & 0.008 \\
\hline Adjusted model $\mathrm{I}^{\mathrm{b}}$ & 1.00 (ref) & $0.66(0.5 \mathrm{I}, 0.86)$ & $0.77(0.60,0.99)$ & $0.70(0.54,0.90)$ & 0.007 \\
\hline Adjusted model $2^{c}$ & 1.00 (ref) & $0.70(0.53,0.91)$ & $0.80(0.62,1.03)$ & $0.73(0.56,0.95)$ & 0.033 \\
\hline Adjusted model $3^{\mathrm{d}}$ & 1.00 (ref) & $0.70(0.54,0.92)$ & $0.80(0.62,1.04)$ & $0.75(0.58,0.98)$ & 0.050 \\
\hline
\end{tabular}

Notes: ${ }^{a} p$ value was obtained by using logistic regression analysis, and data were shown as odds ratios and $95 \%$ confidence interval; ${ }^{b}$ Adjusted for sex (male, female), age (continuous variable), ethnicity (Han ethnicity, others), and only child (yes, no); 'Adjusted for model I plus smoking status (never, former, current), alcohol use (yes, no),

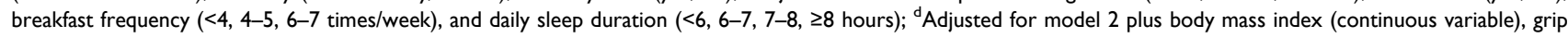
strength (continuous variable), and number of metabolic syndrome components. 
Table 3 Sex-Specific Association Between Total Physical Activity Levels and Depressive Symptoms in College Freshmen ( $\mathrm{n}=\mathbf{2 8 2 0}$ )

\begin{tabular}{|c|c|c|c|c|c|}
\hline \multirow[t]{2}{*}{ Males } & \multicolumn{5}{|c|}{ Quartiles of Total Physical Activity Levels } \\
\hline & QI, n=174 & $Q 2, n=163$ & Q3, $n=195$ & $Q 4, n=167$ & $p$ value $^{a}$ \\
\hline Depressive symptoms, no. & 34 & 19 & 38 & 28 & \\
\hline Non-adjusted model & 1.00 (ref) & $0.54(0.30,1.00)$ & $1.00(0.60,1.67)$ & $0.83(0.48,1.44)$ & 0.182 \\
\hline Adjusted model $\mathrm{I}^{\mathrm{b}}$ & 1.00 (ref) & $0.55(0.30,1.02)$ & $1.03(0.61,1.72)$ & $0.83(0.48,1.45)$ & 0.184 \\
\hline Adjusted model $2^{c}$ & $\mathrm{I} .00$ (ref) & $0.57(0.3 \mathrm{I}, \mathrm{I} .07)$ & $0.99(0.58,1.70)$ & $0.82(0.46,1.45)$ & 0.278 \\
\hline Adjusted model $3^{\mathrm{d}}$ & 1.00 (ref) & $0.55(0.29,1.04)$ & $1.02(0.60,1.76)$ & $0.85(0.48,1.52)$ & 0.223 \\
\hline Females & QI, n=525 & Q2, $n=547$ & Q3, $n=508$ & Q4, $n=54 I$ & \\
\hline Depressive symptoms, no. & 135 & 105 & 99 & 102 & \\
\hline Non-adjusted model & 1.00 (ref) & $0.69(0.5 \mathrm{I}, 0.92)$ & $0.70(0.52,0.94)$ & $0.67(0.50,0.90)$ & 0.017 \\
\hline Adjusted model I ${ }^{\mathrm{b}}$ & 1.00 (ref) & $0.66(0.52,0.92)$ & $0.77(0.53,0.95)$ & $0.70(0.49,0.89)$ & 0.017 \\
\hline Adjusted model $2^{\mathrm{c}}$ & 1.00 (ref) & $0.73(0.54,0.98)$ & $0.74(0.55,1.00)$ & $0.70(0.52,0.94)$ & 0.064 \\
\hline Adjusted model $3^{d}$ & 1.00 (ref) & $0.74(0.55,1.00)$ & $0.74(0.54,1.00)$ & $0.72(0.53,0.97)$ & 0.083 \\
\hline
\end{tabular}

Notes: ${ }^{a} p$ for linear trend was obtained by using logistic regression analysis, and data were shown as odds ratios and $95 \%$ confidence interval; ${ }^{b}$ Adjusted for age (continuous variable), ethnicity (Han ethnicity, others), and only child (yes, no); 'Adjusted for model I plus smoking status (never, former, current), alcohol use (yes, no), breakfast frequency ( $<4,4-5,6-7$ times/week), and daily sleep duration $\left(<6,6-7,7-8, \geq 8\right.$ hours); ${ }^{\mathrm{d}}$ Adjusted for model 2 plus body mass index (continuous variable), grip strength (continuous variable), and number of metabolic syndrome components.

The trend was significant and similar after adjusting all potential covariates ( $p$ for trend $=0.050$ ), but as the number of adjusted covariates increased, the significance gradually decreased. Regarding the sex-specific association between total physical activity levels and depressive symptoms shown in Table 3, the relationship between physical activity level and depressive symptoms in males was insignificant in the univariate model ( $p$ for trend $=0.182$ ), while higher physical activity level was significantly associated with a lower prevalence of depressive symptoms in females ( $p$ for trend $=$ 0.017).

\section{Association Between Physical Activity Intensity and Depressive Symptoms}

Table 4 shows the relationship between physical activity intensity and depressive symptoms before and after adjusting for covariates. There was a significant association between low-intensity physical activity and depressive symptoms after adjusting all covariates ( $p$ for trend $=0.037$ ). However, this result was not seen among those who engaged in moderate-intensity $(p$ for trend $=0.461$ ) and vigorous-intensity physical activity ( $p$ for trend $=$ 0.608). Similarly, the sex-specific analysis showed that low-intensity physical activity levels were inversely associated with depressive symptoms in female students (Table 6). There was no significant correlation between physical activity intensity and depressive symptoms in male students (Table 5).

\section{Discussion}

In this cross-sectional study, we investigated the relationship between physical activity and depressive symptoms among college freshmen. Results indicated that students with higher total physical activity levels tended to have a lower prevalence of depressive symptoms after adjusting for all potential confounding factors. Moreover, higher low-intensity physical activity level is associated with a lower prevalence of depressive symptoms. All these observations showed gender differences.

Many studies supporting that a higher level of physical activity is inversely associated with depressive symptoms, ${ }^{26,27}$ which was consistent with our results. A follow-up study of 2,134 college students showed that insufficient physical activity was significantly associated

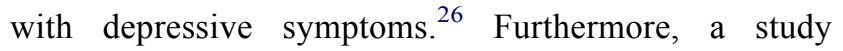
including 43,499 college students indicated that physically active students experienced lower rates of depressive symptoms. ${ }^{27}$ Similar to our study, the results of these two large-scale studies show a weak correlation between physical activity level and depressive symptoms, suggesting that increased physical activity engagement contributes to lower the prevalence of depressive symptoms in youth. On the other hand, previous findings on the relationship between physical activity intensity and depressive symptoms were inconsistent. Motl et al reported that low-intensity exercise is effective in improving depressive symptoms among sedentary older adults, ${ }^{28}$ which supported the results of the current 
Table 4 Association Between Physical Activity Intensity and Depressive Symptoms in College Freshmen $(n=2820)$

\begin{tabular}{|c|c|c|c|c|c|}
\hline \multirow{2}{*}{$\begin{array}{l}\text { Physical Activity Intensity } \\
\text { Low intensity }\end{array}$} & \multicolumn{5}{|c|}{ Quartiles of Physical Activity at Different Intensity Levels } \\
\hline & QI, $n=773$ & Q2, n=774 & Q3, $n=65 I$ & Q4, $n=622$ & $p$ value ${ }^{a}$ \\
\hline Range, METs hour/week & $<9.9$ & $9.9-23.1$ & $23.2-46.2$ & $>46.2$ & \\
\hline Depressive symptoms, no. & 184 & 138 & $|2|$ & 117 & \\
\hline Non-adjusted model & 1.00 (ref) & $0.70(0.54,0.89)$ & $0.73(0.57,0.95)$ & $0.74(0.57,0.96)$ & 0.014 \\
\hline Adjusted model I ${ }^{\mathrm{b}}$ & $1.00(\mathrm{ref})$ & $0.70(0.54,0.89)$ & $0.72(0.55,0.93)$ & $0.72(0.55,0.93)$ & 0.010 \\
\hline Adjusted model $2^{c}$ & 1.00 (ref) & $0.71(0.56,0.92)$ & $0.77(0.59,1.00)$ & $0.74(0.57,0.97)$ & 0.035 \\
\hline Adjusted model $3^{d}$ & 1.00 (ref) & $0.7 \mid(0.55,0.91)$ & $0.77(0.59,1.01)$ & $0.75(0.57,0.98)$ & 0.037 \\
\hline Moderate intensity & $Q I, n=73 I$ & $Q 2, n=665$ & Q3, n=730 & Q4, n=694 & \\
\hline Range, METs hour/week & $<3.3$ & $3.3-9.3$ & $9.4-26.0$ & $>26.0$ & \\
\hline Depressive symptoms, no. & 168 & 127 & 133 & 132 & \\
\hline Non-adjusted model & 1.00 (ref) & $0.79(0.6 \mathrm{I}, \mathrm{I} .03)$ & $0.75(0.58,0.96)$ & $0.79(0.6 \mathrm{I}, \mathrm{I} .02)$ & 0.100 \\
\hline Adjusted model $\mathrm{I}^{\mathrm{b}}$ & 1.00 (ref) & $0.79(0.61,1.03)$ & $0.76(0.59,0.98)$ & $0.79(0.61,1.02)$ & 0.121 \\
\hline Adjusted model $2^{c}$ & 1.00 (ref) & $0.87(0.67,1.13)$ & $0.79(0.6 \mathrm{I}, \mathrm{I} .02)$ & $0.86(0.66,1.12)$ & 0.334 \\
\hline Adjusted model $3^{d}$ & $\mathrm{I} .00$ (ref) & $0.89(0.68,1.16)$ & $0.81(0.62,1.05)$ & $0.88(0.68,1.14)$ & 0.461 \\
\hline Vigorous intensity & $Q I, n=833$ & $Q 2, n=673$ & $Q 3, n=655$ & $Q 4, n=659$ & \\
\hline Range, METs hour/week & $<0.1$ & $0.1-12.0$ & $12.1-32.0$ & $>32.0$ & \\
\hline Depressive symptoms, no. & 169 & 126 & 126 & 139 & \\
\hline Non-adjusted model & 1.00 (ref) & $0.91(0.70,1.17)$ & $0.94(0.72,1.21)$ & $\mathrm{I} .05(0.82, \mathrm{I} .35)$ & 0.698 \\
\hline Adjusted model $\mathrm{I}^{\mathrm{b}}$ & $\mathrm{I} .00$ (ref) & $0.92(0.71,1.19)$ & $0.96(0.74,1.24)$ & $1.10(0.85,1.42)$ & 0.596 \\
\hline Adjusted model $2^{c}$ & $1.00(\mathrm{ref})$ & $0.97(0.75,1.27)$ & $0.99(0.76,1.29)$ & $1.12(0.87,1.46)$ & 0.727 \\
\hline Adjusted model $3^{d}$ & 1.00 (ref) & $0.99(0.76,1.29)$ & $1.01(0.77,1.31)$ & $1.17(0.90,1.51)$ & 0.608 \\
\hline
\end{tabular}

Notes: ${ }^{a} p$ for linear trend was obtained by using logistic regression analysis, and data were shown as odds ratios and $95 \%$ confidence interval; ${ }^{b}$ Adjusted for sex (male, female), age (continuous variable), ethnicity (Han ethnicity, others), and only child (yes, no); 'Adjusted for model I plus smoking status (never, former, current), alcohol use (yes, no), breakfast frequency $\left(<4,4-5,6-7\right.$ times/week), and daily sleep duration $\left(<6,6-7,7-8, \geq 8\right.$ hours); ${ }^{\mathrm{d}}$ Adjusted for model 2 plus body mass index (continuous variable), grip strength (continuous variable), and number of metabolic syndrome components.

Abbreviation: METs, metabolic equivalents.

study. However, some other studies have confirmed that moderate-to-high intensity physical activity, rather than low-intensity, is associated with depressive symptoms. ${ }^{29,30}$ It can be considered that the reason for this discrepancy may be due to the inconsistency in the assessment of physical activity intensity and lack of control of potential confounding factors. First, these previous studies evaluated physical activity intensity by self-reported feeling of fatigue ${ }^{29}$ or walking speed, ${ }^{31}$ which was different from the assessment method by the standardized scale of physical activity in our study. Our evaluation of physical activity intensity took into account the effects of duration and frequency of physical activity, which helps to improve the objectivity and comprehensiveness of physical activity intensity assessment. Moreover, another study by Cahuas et $\mathrm{al}^{30}$ used the same evaluation method of physical activity intensity, but some potential confounding factors, such as sociodemography, lifestyle, and health status, were not controlled. Thus, it is necessary to standardize and unify the evaluation methods of variables and analysis models in future work to get better comparability.

In addition, there was a gender difference in observed findings of this study, with no significant association of total physical activity level and physical activity intensity with depressive symptoms. In fact, physical activity is an important factor in improving body shape, ${ }^{32}$ which may contribute to a lower prevalence of depressive symptoms. It is possible that young women, who are more aware of body shape than men, experience more emotional improvement and stress relief. For instance, body image concern existed to a certain extent in males and females of college students, notably in females. ${ }^{33}$ Studies found that body image dissatisfaction may be contributors for depressive symptoms. ${ }^{34,35}$ Thus, considering that college females 
Table 5 Association Between Physical Activity Intensity and Depressive Symptoms in Male College Freshmen ( $n=699)$

\begin{tabular}{|c|c|c|c|c|c|}
\hline \multirow{2}{*}{$\begin{array}{l}\text { Physical Activity Intensity } \\
\text { Low intensity }\end{array}$} & \multicolumn{5}{|c|}{ Quartiles of Physical Activity at Different Density Levels } \\
\hline & QI, n=224 & Q2, n=220 & Q3, n= 144 & Q4, n=III & $p$ value $^{a}$ \\
\hline Range, METs hour/week & $<9.9$ & $9.9-23.1$ & $23.2-46.2$ & $>46.2$ & \\
\hline Depressive symptoms, no. & 43 & 33 & 26 & 17 & \\
\hline Non-adjusted model & 1.00 (ref) & $0.74(0.45,1.22)$ & $0.93(0.54,1.59)$ & $0.76(0.4 I, I .4 I)$ & 0.633 \\
\hline Adjusted model I ${ }^{\mathrm{b}}$ & $1.00(\mathrm{ref})$ & $0.77(0.46,1.26)$ & $0.94(0.55,1.62)$ & $0.76(0.41,1.40)$ & 0.674 \\
\hline Adjusted model $2^{c}$ & $1.00(\mathrm{ref})$ & $0.77(0.46,1.29)$ & $0.97(0.55,1.69)$ & $0.80(0.43,1.5 \mathrm{I})$ & 0.736 \\
\hline Adjusted model $3^{d}$ & 1.00 (ref) & $0.76(0.45,1.27)$ & $0.97(0.55,1.71)$ & $0.82(0.43,1.55)$ & 0.721 \\
\hline Moderate intensity & $Q 1, n=165$ & $Q 2, n=161$ & Q3, n=197 & $Q 4, n=176$ & \\
\hline Range, METs hour/week & $<3.3$ & $3.3-9.3$ & $9.4-26.0$ & $>26.0$ & \\
\hline Depressive symptoms, no. & 35 & 23 & 28 & 33 & \\
\hline Non-adjusted model & $\mathrm{I} .00$ (ref) & $0.62(0.35,1.10)$ & $0.62(0.36,1.06)$ & $0.86(0.50,1.46)$ & 0.227 \\
\hline Adjusted model I ${ }^{\mathrm{b}}$ & $1.00(\mathrm{ref})$ & $0.61(0.35,1.06)$ & $0.61(0.36,1.06)$ & $0.84(0.49,1.43)$ & 0.228 \\
\hline Adjusted model $2^{c}$ & $\mathrm{I} .00$ (ref) & $0.64(0.35,1.16)$ & $0.61(0.35,1.07)$ & $0.86(0.49,1.49)$ & 0.265 \\
\hline Adjusted model $3^{d}$ & 1.00 (ref) & $0.70(0.38,1.29)$ & $0.73(0.40,1.32)$ & $1.54(0.70,3.39)$ & 0.114 \\
\hline Vigorous intensity & QI, n=I49 & Q2, $n=156$ & Q3, n= 177 & $Q 4, n=217$ & \\
\hline Range, METs hour/week & $<0.1$ & $0.1-12.0$ & $12.1-32.0$ & $>32.0$ & \\
\hline Depressive symptoms, no. & 25 & 25 & 23 & 46 & \\
\hline Non-adjusted model & 1.00 (ref) & $0.95(0.52,1.74)$ & $0.74(0.40,1.37)$ & $1.33(0.75,1.34)$ & 0.190 \\
\hline Adjusted model I ${ }^{\mathrm{b}}$ & 1.00 (ref) & $0.94(0.5 \mathrm{I}, \mathrm{I} .73)$ & $0.74(0.40,1.37)$ & $1.35(0.79,2.33)$ & 0.175 \\
\hline Adjusted model $2^{\mathrm{C}}$ & $\mathrm{I} .00$ (ref) & $1.09(0.58,2.05)$ & $0.7 \mathrm{I}(0.38,1.34)$ & $1.38(0.79,2.42)$ & 0.149 \\
\hline Adjusted model $3^{d}$ & 1.00 (ref) & $1.19(0.63,2.24)$ & $0.75(0.39,1.42)$ & $1.54(0.87,2.72)$ & 0.092 \\
\hline
\end{tabular}

Notes: ${ }^{a} p$ for linear trend was obtained by using logistic regression analysis, and data were shown as odds ratios and $95 \%$ confidence interval; ${ }^{b}$ Adjusted for sex (male, female), age (continuous variable), ethnicity (Han ethnicity, others), and only child (yes, no); ${ }^{\mathrm{C} A d j u s t e d ~ f o r ~ m o d e l ~ I ~ p l u s ~ s m o k i n g ~ s t a t u s ~(n e v e r, ~ f o r m e r, ~ c u r r e n t), ~ a l c o h o l ~ u s e ~}$ (yes, no), breakfast frequency (<4, 4-5, 6-7 times/week), and daily sleep duration (<6, 6-7, 7-8, $\geq 8$ hours); ${ }^{\mathrm{d} A d j u s t e d ~ f o r ~ m o d e l ~} 2$ plus body mass index (continuous variable), grip strength (continuous variable), and number of metabolic syndrome components.

Abbreviation: METs, metabolic equivalents.

may have more satisfaction with body image than males, young women may gain more mental health benefits from physical activity. Furthermore, it is also important to note that male participants only made up nearly a quarter of the total sample size, the limited sample size may be another factor leading to insignificant results between physical activity intensity and depressive symptoms in male students.

The mechanism of depressive symptoms is not fully understood, but some biological mechanisms and psychological factors are recognized. ${ }^{36}$ Studies have shown that chronic inflammation throughout the body may contribute to depressive symptoms. ${ }^{37}$ A study of 73,131 people found that increased C-reactive protein levels were associated with an increased risk of psychological distress and depression in cross-sectional analysis and were also associated with an increased depression hospitalization risk in a prospective analysis. ${ }^{38}$ In addition, neuroinflammation, especially microglia activation, may play an important role in the development of depressive symptoms. ${ }^{39}$ Reichenberg et al found a temporary increase in anxiety and depression in healthy men by injecting them with Salmonella abortus equi endotoxin to activate microglia. ${ }^{40}$ Thus, both peripheral inflammation and neuroinflammation have a certain effect on depressive symptoms, while exercise plays a significant role in reducing inflammation. A meta-analysis showed that exercise reduced the C-reactive protein levels regardless of an individual's age and gender, and this effect was more pronounced in people with a lower BMI or body fat percentage. ${ }^{41}$ Also, a study in mice with neuropathic pain found that 2 weeks of low-intensity exercise inhibited peripheral and central nervous system inflammation. ${ }^{42}$ The vascular disease also may affect depressive symptoms, especially the presence of focal vascular lesions and high white matter signalling. ${ }^{43}$ A cross-sectional and longitudinal study on the relationship between senile depression and white matter 
Table 6 Association Between Physical Activity Intensity and Depressive Symptoms in Female College Freshmen ( $n=2121$ )

\begin{tabular}{|c|c|c|c|c|c|}
\hline \multirow{2}{*}{$\begin{array}{l}\text { Physical Activity Intensity } \\
\text { Low intensity }\end{array}$} & \multicolumn{5}{|c|}{ Quartiles of Physical Activity at Different Density Levels } \\
\hline & QI, n=549 & Q2, n=554 & Q3, n=507 & Q4, n=5 I I & $p$ value $^{a}$ \\
\hline Range, METs hour/week & $<9.9$ & $9.9-23.1$ & $23.2-46.2$ & $>46.2$ & \\
\hline Depressive symptoms, no. & $|4|$ & 105 & 95 & 110 & \\
\hline Non-adjusted model & $\mathrm{I} .00$ (ref) & $0.68(0.5 \mathrm{I}, 0.90)$ & $0.67(0.50,0.90)$ & $0.70(0.53,0.94)$ & 0.013 \\
\hline Adjusted model $\mathrm{I}^{\mathrm{b}}$ & $1.00(\mathrm{ref})$ & $0.69(0.52,0.91)$ & $0.66(0.49,0.89)$ & $0.69(0.52,0.93)$ & 0.013 \\
\hline Adjusted model $2^{c}$ & $1.00(\mathrm{ref})$ & $0.71(0.53,0.95)$ & $0.73(0.54,0.98)$ & $0.71(0.53,0.96)$ & 0.050 \\
\hline Adjusted model $3^{d}$ & $\mathrm{I} .00$ (ref) & $0.69(0.52,0.93)$ & $0.72(0.53,0.97)$ & $0.71(0.53,0.96)$ & 0.041 \\
\hline Moderate intensity & $Q I, n=566$ & $Q 2, n=504$ & $Q 3, n=533$ & $Q 4, n=518$ & \\
\hline Range, METs hour/week & $<3.3$ & $3.3-9.3$ & $9.4-26.0$ & $>26.0$ & \\
\hline Depressive symptoms, no. & 133 & 104 & 105 & 99 & \\
\hline Non-adjusted model & 1.00 (ref) & $0.85(0.63,1.13)$ & $0.80(0.60,1.07)$ & $0.77(0.57,1.03)$ & 0.285 \\
\hline Adjusted model $\mathrm{I}^{\mathrm{b}}$ & $\mathrm{I} .00$ (ref) & $0.86(0.64,1.15)$ & $0.81(0.60,1.08)$ & $0.77(0.57,1.03)$ & 0.292 \\
\hline Adjusted model $2^{c}$ & $\mathrm{I} .00(\mathrm{ref})$ & $0.95(0.70,1.27)$ & $0.83(0.62,1.12)$ & $0.84(0.62,1.13)$ & 0.534 \\
\hline Adjusted model $3^{d}$ & 1.00 (ref) & $0.97(0.72,1.30)$ & $0.87(0.64,1.18)$ & $0.97(0.64,1.47)$ & 0.826 \\
\hline Vigorous intensity & QI, n=684 & $Q 2, n=517$ & Q3, n=478 & Q4, $n=442$ & \\
\hline Range, METs hour/week & $<0.1$ & $0.1-12.0$ & $12.1-32.0$ & $>32.0$ & \\
\hline Depressive symptoms, no. & 144 & 101 & 103 & 93 & \\
\hline Non-adjusted model & $\mathrm{I} .00$ (ref) & $0.91(0.69, \mid .21)$ & $1.03(0.77,1.37)$ & $\mathrm{I} .00(0.75, \mathrm{I} .34)$ & 0.872 \\
\hline Adjusted model I ${ }^{\mathrm{b}}$ & $\mathrm{I} .00$ (ref) & $0.91(0.68,1.21)$ & $1.02(0.77,1.36)$ & $0.99(0.74,1.33)$ & 0.888 \\
\hline Adjusted model $2^{c}$ & $\mathrm{I} .00$ (ref) & $0.95(0.7 \mathrm{I}, \mathrm{I} .28)$ & $1.06(0.80,1.43)$ & $1.01(0.75,1.36)$ & 0.924 \\
\hline Adjusted model $3^{d}$ & $1.00(\mathrm{ref})$ & $0.95(0.7 \mathrm{I}, \mathrm{I} .28)$ & $1.08(0.81,1.45)$ & $1.03(0.76,1.40)$ & 0.888 \\
\hline
\end{tabular}

Notes: ${ }^{a} p$ for linear trend was obtained by using logistic regression analysis, and data were shown as odds ratios and $95 \%$ confidence interval; ${ }^{b}$ Adjusted for sex (male, female), age (continuous variable), ethnicity (Han ethnicity, others), and only child (yes, no); 'Adjusted for model I plus smoking status (never, former, current), alcohol use (yes, no), breakfast frequency (<4, 4-5, 6-7 times/week), and daily sleep duration $\left(<6,6-7,7-8, \geq 8\right.$ hours); ${ }^{\mathrm{d}}$ Adjusted for model 2 plus body mass index (continuous variable), grip strength (continuous variable), and number of metabolic syndrome components.

Abbreviation: METs, metabolic equivalents.

integrity found that decreased white matter integrity is associated with advanced depression and predicts future symptoms. ${ }^{44}$ Nevertheless, six months of biking was associated with increased white matter integrity in healthy people. ${ }^{45}$ These previous reports support our findings that higher levels of physical activity were associated with less depressive symptoms.

The present study has some limitations. First, the physical activity measurements were obtained by selfreport questionnaire, which may lead to recall bias. Objective measured physical activity by the accelerometer may better reflect the actual level of physical activity. Second, the data are from cross-sectional surveys, so we can not determine the causal relationship between physical activity and depressive symptoms. Third, our sample is limited to Chinese college freshmen, and it is not clear whether the findings apply to other populations.

\section{Conclusion}

In conclusion, our study found that high total physical activity levels are associated with a lower prevalence of depressive symptoms in Chinese college freshmen. More participation in low-intensity physical activity may contribute to a lower prevalence of depressive symptoms. These findings revealed the importance of physical activity participation in mental health promotion in youth. Future prospective and experimental studies will be needed to confirm the relationship between physical activity intensity and depressive symptoms.

\section{Abbreviations}

BMI, body mass index; CI, confidence interval; IPAQSF, International Physical Activity Questionnaire-Short Format; METs, metabolic equivalents; MetS, metabolic syndrome; ORs, odds ratios; SDS, Self-rating Depression Scale. 


\section{Data Sharing Statement}

The datasets used and/or analyzed during the current study are available from the corresponding author upon reasonable request.

\section{Ethics Approval and Consent to Participate}

All procedures performed in this study were under the ethical standards of the institutional research committee and the 1964 Helsinki Declaration and its later amendments or comparable ethical standards. The study protocol was approved by the Ethics Committee of the College of Education, Zhejiang University. Informed consent was obtained from individual participants included in the study.

\section{Acknowledgments}

The authors are extremely grateful to all the participants of this study. The authors would also like to express their sincere gratitude to the staff of the College of Sports Science, Shenyang Normal University.

\section{Author Contributions}

All authors contributed to data analysis, drafting or revising the article, have agreed on the journal to which the article will be submitted, gave final approval of the version to be published, and agree to be accountable for all aspects of the work.

\section{Funding}

The present study was partially supported by the "Hundred Talents Program" funding from Zhejiang University (188020*194221802/004/001).

\section{Disclosure}

The authors declared no potential conflicts of interest with respect to the research, authorship, and/or publication of this article.

\section{References}

1. Huang Y, Wang Y, Wang H, et al. Prevalence of mental disorders in China: a cross-sectional epidemiological study. Lancet Psychiatry. 2019;6(3):211-224. doi:10.1016/S2215-0366(18)30511-X

2. World Health Organization. Depression and Other Common Mental Disorders: Global Health Estimates. Geneva: World Health Organization; 2017.

3. Garlow SJ, Rosenberg J, Moore JD, et al. Depression, desperation, and suicidal ideation in college students: results from the American foundation for suicide prevention college screening project at Emory University. Depress Anxiety. 2008;25(6):482-488. doi:10.1002/ da. 20321
4. United Nations. Youth. United nations. Available from: https://www. un.org/en/sections/issues-depth/youth-0/. Accessed August 14, 2020.

5. Steptoe A, Tsuda A, Tanaka Y, Wardle J. Depressive symptoms, socio-economic background, sense of control, and cultural factors in university students from 23 countries. Int J Behav Med. 2007;14 (2):97-107. doi:10.1007/BF03004175

6. Meng RW, Yu CQ, Liu N, et al. Association of depression with all-cause and cardiovascular disease mortality among adults in China. JAMA Network Open. 2020;3(2):e1921043. doi:10.1001/ jamanetworkopen.2019.21043

7. Lei XY, Xiao LM, Liu YN, Li YM. Prevalence of depression among Chinese university students: a meta-analysis. PLoS One. 2016;11(4): e0153454. doi:10.1371/journal.pone.0153454

8. Demirci K, Akgonul M, Akpinar A. Relationship of smartphone use severity with sleep quality, depression, and anxiety in university students. J Behav Addict. 2015;4(2):85-92. doi:10.1556/2006.4.2015.010

9. Woods HC, Scott H. \#Sleepyteens: social media use in adolescence is associated with poor sleep quality, anxiety, depression and low selfesteem. $J$ Adolescence. 2016;51:41-49. doi:10.1016/j. adolescence.2016.05.008

10. Byeon H. Association between weight misperception patterns and depressive symptoms in Korean young adolescents: national cross-sectional study. PLoS One. 2015;10(8):e0131322. doi:10.1371/journal.pone.0131322

11. Xu Y, Qi J, Yang Y, Wen X. The contribution of lifestyle factors to depressive symptoms: a cross-sectional study in Chinese college students. Psychiatry Res. 2016;245:243-249. doi:10.1016/j. psychres.2016.03.009

12. McMahon EM, Corcoran P, O'Regan G, et al. Physical activity in European adolescents and associations with anxiety, depression and well-being. Eur Child Adolesc Psychiatry. 2017;26(1):111-122. doi:10.1007/s00787-016-0875-9

13. Hong X, Li JQ, Xu F, et al. Physical activity inversely associated with the presence of depression among urban adolescents in regional China. BMC Public Health. 2009;9:148.

14. Tsujita N, Akamatsu Y, Nishida MM, Hayashi T, Moritani T. Physical activity, nutritional status, and autonomic nervous system activity in healthy young adults with higher levels of depressive symptoms and matched controls without depressive symptoms: a cross-sectional study. Nutrients. 2020;12(3):690. doi:10.3390/nu12030690

15. Viana RB, Gentil P, Naves JPA, et al. Interval training improves depressive symptoms but not anxious symptoms in healthy women. Front Psychiatry. 2019;10:661. doi:10.3389/fpsyt.2019.00661

16. Paolucci EM, Loukov D, Bowdish DME, Heisz JJ. Exercise reduces depression and inflammation but intensity matters. Biol Psychol. 2018;133:79-84. doi:10.1016/j.biopsycho.2018.01.015

17. Choi HY, Han HJ, Choi JW, Jung HY, Joa KL. Superior effects of high-intensity interval training compared to conventional therapy on cardiovascular and psychological aspects in myocardial infarction. Ann Rehabil Med. 2018;42(1):145-153. doi:10.5535/arm.2018.42.1.145

18. Olson RL, Brush CJ, Ehmann PJ, Alderman BL. A randomized trial of aerobic exercise on cognitive control in major depression. Clin Neurophysiol. 2017;128(6):903-913. doi:10.1016/j.clinph.2017.01.023

19. Guo F, Tian Y, Cui Y, Huang C. Night-eating syndrome and depressive symptoms in college freshmen: fitness improvement tactics in youths (FITYou) project. Psychol Res Behav Manag. 2020;13:185. doi:10.2147/PRBM.S234025

20. Zhang Z, Tian Y, Zhong F, et al. Association between oral health-related quality of life and depressive symptoms in Chinese college students: fitness improvement tactics in youths (FITYou) project. Health Qual Life Outcomes. 2019;17(1):96. doi:10.1186/ s12955-019-1163-4

21. World Medical Association. World medical association declaration of Helsinki: ethical principles for medical research involving human subjects. JAMA. 2013;310(20):2191-2194. doi:10.1001/ jama.2013.281053 
22. Zung WWK. A self-rating depression scale. Arch Gen Psychiatry. 1965;12(1):63-70. doi:10.1001/archpsyc.1965.01720310065008

23. Dunstan DA, Scott N. Clarification of the cut-off score for Zung's self-rating depression scale. BMC Psychiatry. 2019;19(1):177. doi:10.1186/s12888-019-2161-0

24. Craig CL, Marshall AL, Sjöström M, et al. International physical activity questionnaire: 12-country reliability and validity. Med Sci Sports Exerc. 2003;35(8):1381-1395. doi:10.1249/01.MSS.0000078924.61453.FB

25. Alberti KG, Eckel RH, Grundy SM, et al. Harmonizing the metabolic syndrome: a joint interim statement of the international diabetes federation task force on epidemiology and prevention; National heart, lung, and blood institute; American heart association; world heart federation; International atherosclerosis society; and International association for the study of obesity. Circulation. 2009;120(16):1640-1645. doi:10.1161/ CIRCULATIONAHA.109.192644

26. Xie HB, Tao SM, Zhang YK, Tao FB, Wu XY. Impact of problematic mobile phone use and insufficient physical activity on depression symptoms: a college-based follow-up study. BMC Public Health. 2019;19(1):1640. doi:10.1186/s12889-019-7873-z

27. Taliaferro LA, Rienzo BA, Pigg RM, Miller MD, Dodd VJ. Associations between physical activity and reduced rates of hopelessness, depression, and suicidal behavior among college students. $J \mathrm{Am}$ Coll Health. 2009;57(4):427-436. doi:10.3200/JACH.57.4.427-436

28. Motl RW, Konopack JF, McAuley E, Elavsky S, Jerome GJ, Marquez DX. Depressive symptoms among older adults: long-term reduction after a physical activity intervention. J Behav Med. 2005;28 (4):385. doi:10.1007/s10865-005-9005-5

29. Harbour VJ, Behrens TK, Kim HS, Kitchens CL. Vigorous physical activity and depressive symptoms in college students. J Phys Act Health. 2008;5(4):516-526. doi:10.1123/jpah.5.4.516

30. Cahuas A, He Z, Zhang Z, Chen W. Relationship of physical activity and sleep with depression in college students. J Am Coll Health. 2019;68:557-564.

31. Varma VR, Tan EJ, Wang T, et al. Low-intensity walking activity is associated with better health. J Appl Gerontol. 2014;33(7):870-887. doi:10.1177/0733464813512896

32. Hausenblas HA, Fallon E. Exercise and body image: a meta-analysis. Psychol Health. 2006;21(1):33-47. doi:10.1080/14768320500105270

33. El Ansari W, Dibba E, Labeeb S, Stock C. Body image concern and its correlates among male and female undergraduate students at Assuit University in Egypt. Glob J Health Sci. 2014;6(5):105-117. doi:10.5539/gjhs.v6n5p105

34. Friedman KE, Reichmann SK, Costanzo PR, Musante GJ. Body image partially mediates the relationship between obesity and psychological distress. Obes Res. 2002;10(1):33-41. doi:10.1038/ oby. 2002.5
35. Gavin AR, Simon GE, Ludman EJ. The association between obesity, depression, and educational attainment in women: the mediating role of body image dissatisfaction. J Psychosom Res. 2010;69 (6):573-581. doi:10.1016/j.jpsychores.2010.05.001

36. Dean J, Keshavan M. The neurobiology of depression: an integrated view. Asian $J$ Psychiatr. 2017;27:101-111. doi:10.1016/j. ajp.2017.01.025

37. Penninx BW, Milaneschi Y, Lamers F, Vogelzangs N. Understanding the somatic consequences of depression: biological mechanisms and the role of depression symptom profile. BMC Med. 2013;11(1):129. doi:10.1186/1741-7015-11-129

38. Wium-Andersen MK, Ørsted DD, Nielsen SF, Nordestgaard BG. Elevated C-reactive protein levels, psychological distress, and depression in 73131 individuals. JAMA Psychiatry. 2013;70 (2):176-184. doi:10.1001/2013.jamapsychiatry.102

39. Brites D, Fernandes A. Neuroinflammation and depression: microglia activation, extracellular microvesicles and microRNA dysregulation. Front Cell Neurosci. 2015;9:476.

40. Reichenberg A, Yirmiya R, Schuld A, et al. Cytokine-associated emotional and cognitive disturbances in humans. Arch Gen Psychiatry. 2001;58(5):445-452. doi:10.1001/archpsyc.58.5.445

41. Fedewa MV, Hathaway ED, Ward-Ritacco CL. Effect of exercise training on $\mathrm{C}$ reactive protein: a systematic review and meta-analysis of randomised and non-randomised controlled trials. Br J Sports Med. 2017;51(8):670-676. doi:10.1136/bjsports-2016095999

42. Bobinski F, Teixeira JM, Sluka KA, Santos ARS. Interleukin-4 mediates the analgesia produced by low-intensity exercise in mice with neuropathic pain. Pain. 2018;159(3):437-450. doi:10.1097/j. pain.0000000000001109

43. Taylor WD, Aizenstein HJ, Alexopoulos GS. The vascular depression hypothesis: mechanisms linking vascular disease with depression. Mol Psychiatry. 2013;18(9):963-974.

44. Reppermund S, Zhuang L, Wen W, et al. White matter integrity and late-life depression in community-dwelling individuals: diffusion tensor imaging study using tract-based spatial statistics. $\mathrm{Br}$ J Psychiatry. 2014;205(4):315-320. doi:10.1192/bjp.bp.113.142109

45. Svatkova A, Mandl RC, Scheewe TW, Cahn W, Kahn RS, Hulshoff Pol HE. Physical exercise keeps the brain connected: biking increases white matter integrity in patients with schizophrenia and healthy controls. Schizophr Bull. 2015;41(4):869-878. doi:10.1093/schbul/ sbv033
Psychology Research and Behavior Management

\section{Publish your work in this journal}

Psychology Research and Behavior Management is an international, peer-reviewed, open access journal focusing on the science of psychology and its application in behavior management to develop improved outcomes in the clinical, educational, sports and business arenas. Specific topics covered in the journal include: Neuroscience, memory and decision making; Behavior modification and management; Clinical applications; Business and sports performance management; Social and developmental studies; Animal studies. The manuscript management system is completely online and includes a very quick and fair peer-review system, which is all easy to use. Visit http://www. dovepress.com/testimonials.php to read real quotes from published authors. 